\title{
The convolutional double copy: a case study with a point
}

\author{
Andrés Luna, ${ }^{a}$ Silvia Nagy ${ }^{b}$ and Chris D. White ${ }^{c}$ \\ ${ }^{a}$ Mani L. Bhaumik Institute for Theoretical Physics, UCLA Department of Physics and Astronomy, \\ Los Angeles, CA 90095, U.S.A. \\ ${ }^{b}$ Centre for Astronomy and Particle Theory, University Park, \\ Nottingham NG7 2RD, U.K. \\ ${ }^{c}$ Centre for Research in String Theory, School of Physics and Astronomy, \\ Queen Mary University of London, \\ 327 Mile End Road, London E1 4NS, U.K. \\ E-mail: luna@physics.ucla.edu, Silvia.Nagy@nottingham.ac.uk, \\ christopher.white@qmul.ac.uk
}

ABSTRACT: The double copy relates scattering amplitudes in gauge and gravity theories. It has also been extended to classical solutions, and a number of approaches have been developed for doing so. One of these involves expressing fields in a variety of (super)gravity theories in terms of convolutions of gauge fields, including also BRST ghost degrees of freedom that map neatly to their corresponding counterparts in gravity. In this paper, we spell out how to use the convolutional double copy to map gauge and gravity solutions in the manifest Lorenz and de Donder gauges respectively. We then apply this to a particular example, namely the point charge in pure gauge theory. As well as clarifying how to use the convolutional approach, our results provide an alternative point of view on a recent discussion concerning whether point charges map to the Schwarzschild solution, or the more general two-parameter JNW solution, which includes a dilaton field. We confirm the latter.

Keywords: BRST Quantization, Gauge Symmetry, Scattering Amplitudes, Space-Time Symmetries

ARXiv EPRINT: 2004.11254 


\section{Contents}

1 Introduction 1

2 From Lorenz to de Donder: a local dictionary 3

$\begin{array}{lll}3 & \text { The JNW solution from a general dictionary } & 7\end{array}$

4 Conclusion $\quad 11$

\section{Introduction}

The BCJ double copy of refs. [1-3] is by now a well-established relationship between scattering amplitudes in gauge and gravity theories. Since its inception, a number of approaches have tried to extend its remit to classical solutions, exact or otherwise. Examples include the use of Kerr-Schild coordinates [4-14], spinorial methods [15, 16], worldline methods [17-21], perturbative diagrammatic reasoning [22-25], and double field theory [26, 27]. In this paper, we will focus on another approach, first introduced in ref. [28], in which the field content of a gravity theory in position space can be obtained using convolutions of fields from a gauge theory. The convolution operation is defined for two functions $f(x)$ and $g(x)$ via

$$
[f \star g](x)=\int d^{4} y f(x) g(x-y) .
$$

This is both commutative and associative, and furthermore obeys the derivative rule

$$
\partial_{\mu}(f \star g)=\left(\partial_{\mu} f\right) \star g=f \star\left(\partial_{\mu} g\right) .
$$

The motivation for this product is that, for scattering amplitudes, the double copy operates via products of functions in momentum space. Upon Fourier transforming back to position space, such products would become the convolutions of eq. (1.1).

A restriction of the convolution approach is that it is currently only defined for linearised gauge and gravity theories. However, the Kerr-Schild double copy of ref. [4] also relates solutions of the linear equations (which happen in that case to be solutions of the full non-linear theory). Furthermore, working at linear level for more general solutions certainly does not prevent the use of the convolution formalism to gain significant insights. It was used in refs. [29-36], for example, to construct a wide catalogue of double copy examples, involving highly exotic (super-)gravity theories. Another significant advantage of the convolution approach is that it works, in principle, for arbitrary gauge choices in both the gauge and gravity theories, a feature which is not typically shared by other classical double copy approaches, or by the original BCJ double copy for amplitudes. The latter relies on a certain duality between colour and kinematics being made manifest [1], which is possible in principle using both gauge transformations and field redefinitions, ${ }^{1}$ collectively

\footnotetext{
${ }^{1}$ See, however, refs. [37, 38], wich describes how the BCJ duality requirement can be relaxed.
} 
known as generalised gauge transformations. We remark that this is not the case for the original KLT double copy construction [39].

In a non-abelian gauge theory, a gauge-fixing condition gives rise to ghost fields according to the usual Faddeev-Popov procedure [40], and the resulting action obeys the wellknown BRST invariance [41-44], where an analogous story holds in (super-)gravity [45, 46]. How to marry the BRST formalism with the double copy was addressed in refs. [47, 48], which extended the convolutional double copy to include ghost fields, such that all physical degrees of freedom in the gravity theory can be obtained by combining both physical and ghost fields from the two chosen gauge theories (see also refs. $[49,50]$ for earlier work describing such a correspondence). It was also used to study the issue of the separation of the dilaton degree of freedom from the trace of the graviton, pointed out in e.g. refs. [22, 51]. The particular dictionary between gauge and gravity fields is not unique, but will depend on the choices of gauge-fixing condition on both the gauge and gravity sides. Although the relevant principles were defined in ref. [47], there have to date been few results in the literature regarding both (i) how to construct the convolutional double copy dictionary explicitly, ${ }^{2}$ and (ii) its consequences for particular solutions. The aim of this paper is to rectify this situation, by carrying out the convolutional double copy for particular classical solutions, with particular gauge choices.

More specifically, we will examine the expression for a point charge in pure (nonsupersymmetric) Yang-Mills theory, and construct its double copy in the relevant gravity theory, namely $\mathcal{N}=0$ Supergravity. The latter consists of General Relativity coupled to a dilaton and axion (two-form) field, and we will choose the commonly-used Lorenz and de Donder gauges in the gauge and gravity theory respectively. There are good reasons to choose this particular example. Firstly, it is arguably the simplest case of a gauge theory solution that one may use to probe the classical double copy. Secondly, there has been some discussion in the recent literature regarding the precise identity of the point charge's double copy. The Kerr-Schild approach of ref. [4] was the first to consider this, and identified the point charge with a Schwarzschild black hole. However, ref. [17] used worldline methods to point out that one typically expects the dilaton field to be turned on in the gravity theory, which is seemingly at odds with ref. [4]. ${ }^{3}$ Reference [22] argued that indeed the most general double copy of the point charge should be regarded as the twoparameter JNW solution [53], consisting of a spherically symmetric graviton and dilaton system. By choosing generalised gauges appropriately, one may select any special case of JNW, including Schwarzschild. This point of view was corroborated by the recent study of ref. [27], using double field theory. We will see that the BRST convolutional double copy provides a highly useful complementary analysis of this situation. We will demonstrate explicitly how the two-parameter JNW solution is obtained, whilst also seeing how the various ghost degrees of freedom can be independently chosen so as to restrict to particular cases. Our results thus clarify the convolutional double copy framework, whilst also exhibiting a topical application.

\footnotetext{
${ }^{2}$ Reference [52] provides a recent pedagogical review of this and many other double-copy-related topics.

${ }^{3}$ Interestingly, the dilaton turns on in three spacetime dimensions, even in the Kerr-Schild approach [11].
} 
The structure of our paper is as follows. In section 2, we describe the convolutional double copy in more detail, and try to construct the simplest possible dictionary between gauge and gravity fields in the Lorenz and de Donder gauges respectively. In this warmup example, we will omit possible terms containing inverse derivatives, which correspond to non-local operators in position space. However, we will see that this prescription is insufficient to obtain the full two parameter JNW solution in de Donder gauge. Thus, in section 3 , we will append the dictionary accordingly, showing explicitly how the point charge and JNW solutions are related. Finally, we discuss our results and conclude in section 4 .

\section{From Lorenz to de Donder: a local dictionary}

In this section, we give our first illustration of the convolutional double copy, by showing how one may construct a dictionary between Yang-Mills and gravity fields, including ghosts (see also refs. [47, 48]). As discussed above, we will first consider only terms containing products of fields, without including inverse derivatives. This will act as a practice run for a more general approach, to be considered in what follows. Also, although the details of this dictionary have appeared before in the literature, it has not been applied to the point charge. We will see below that this has interesting consequences.

We will focus on the case in which both gauge theory solutions entering the double copy are the same. One may then define the circle product of two non-abelian gauge fields [28]:

$$
A_{\mu} \circ A_{\nu} \equiv A_{\mu}^{a} \star \Phi^{a a^{\prime}} \star A_{\nu}^{a^{\prime}}
$$

where $\Phi^{a a^{\prime}}$ is a so-called spectator field, that couples together the adjoint indices $\left(a, a^{\prime}\right)$. This is a scalar field with two adjoint indices, and is related to the biadjoint scalar field that appears in other classical double copy approaches $[4,54-56]{ }^{4}$ A similar product may be used for the (anti)-ghost fields $\left(c^{a}, \bar{c}^{a}\right)$ appearing in the Yang-Mills theory, where from now on we will suppress adjoint indices for brevity, unless otherwise stated.

The first step in applying the convolutional double copy is to write a general ansatz expressing the graviton $h_{\mu \nu}$ and dilaton field $\phi$ in terms of the Yang-Mills fields $\left(A_{\mu}, c, \bar{c}\right):^{5}$

$$
\begin{aligned}
h_{\mu \nu} & =2 A_{\mu} \circ A_{\nu}+\eta_{\mu \nu}\left(a_{1} A^{\rho} \circ A_{\rho}+a_{2} c \circ \bar{c}\right) ; \\
\varphi & =a_{3} A^{\rho} \circ A_{\rho}+a_{4} c \circ \bar{c} .
\end{aligned}
$$

Without loss of generality, we have used an overall normalisation constant on the righthand side of eq. (2.2), to fix a factor of 2 in the first term to match the normalisation for the general asymmetric case [47]. The Yang-Mills action, including ghosts, is invariant under the BRST transformations

$$
Q A_{\mu}=\partial_{\mu} c, \quad Q c=0, \quad Q \bar{c}=\frac{1}{\xi} G\left[A_{\mu}\right],
$$

\footnotetext{
${ }^{4}$ The field $\Phi^{a a^{\prime}}$ as defined here is actually the convolution inverse of the biadjoint scalar field, and corrects for the fact that the biadjoint field appears in $A_{\mu}^{a}$, and thus has been counted twice in eq. (2.1).

${ }^{5}$ The antisymmetric two-form field $B_{\mu \nu}$ can only appear if the two gauge fields are chosen to be different.
} 
where $Q$ is a Grassmann-valued charge,

$$
G[A]=\partial \cdot A
$$

the gauge-fixing condition, which we have chosen to correspond to the Lorenz gauge, and $\xi$ an arbitrary multiplier that defines the usual general family of such covariant gauges. A crucial observation of ref. [47] is that one should identify the BRST operator $Q$ with its counterpart in the gravity theory, which acts on the graviton and dilaton via

$$
Q h_{\mu \nu}=\partial_{\mu} c_{\nu}+\partial_{\nu} c_{\mu}, \quad Q \varphi=0
$$

Here $c_{\mu}$ are the ghosts for the gravity field, and requiring the BRST constraints to be satisfied in the gravity theory allows the parameters entering the dictionary of eqs. (2.2), (2.3) to be constrained. To this end, one may use eq. (2.4) and the (anti-)linearity of the BRST charge

$$
Q[A \circ B]=(Q A) \circ B \pm A \circ(Q B),
$$

where the upper (lower) sign holds if $A$ is bosonic (fermionic), to get

$$
Q h_{\mu \nu}=4 \partial_{(\mu} c \circ A_{\nu)}+\eta_{\mu \nu}\left(2 a_{1}-\frac{a_{2}}{\xi}\right) \partial^{\rho} c \circ A_{\rho},
$$

where we define

$$
a_{(\mu} b_{\nu)}=\frac{1}{2}\left(a_{\mu} b_{\nu}+a_{\nu} b_{\mu}\right)
$$

Consistency with eq. (2.6) immediately gives the constraint

$$
a_{2}=2 \xi a_{1}
$$

and then we can read off

$$
c_{\mu}=2 c \circ A_{\nu}, \quad \Rightarrow \quad \bar{c}_{\mu}=2 \bar{c} \circ A_{\nu}
$$

where the second condition for the anti-ghost follows from conjugation. Similarly, eq. (2.3) yields

$$
Q \varphi=\left(2 a_{3}-\frac{a_{4}}{\xi}\right) \partial^{\rho} c \circ A_{\rho}=0 \quad \Rightarrow \quad a_{4}=2 \xi a_{3} .
$$

At this point our dictionary reads

$$
\begin{aligned}
h_{\mu \nu} & =2 A_{\mu} \circ A_{\nu}+a_{1} \eta_{\mu \nu}\left(A^{\rho} \circ A_{\rho}+2 \xi c \circ \bar{c}\right) \\
\varphi & =a_{3}\left(A^{\rho} \circ A_{\rho}+2 \xi c \circ \bar{c}\right)
\end{aligned}
$$

and we may fix the remaining coefficients by considering the known BRST transformations for the level one (anti)-ghosts:

$$
Q c_{\mu}=0, \quad Q \bar{c}_{\mu}=\frac{1}{\xi} G_{\mu}\left[h_{\mu \nu}, \varphi\right]
$$


where $G_{\mu}\left[h_{\mu \nu}, \varphi\right]$ is the gauge-fixing condition in the gravity theory, ${ }^{6}$ which may involve both the graviton and dilaton fields in general. However, we will take it to correspond to the de Donder gauge:

$$
G_{\mu}=\partial^{\nu} \bar{h}_{\mu \nu}, \quad \bar{h}_{\mu \nu}=h_{\mu \nu}-\frac{h}{2} \eta_{\mu \nu}, \quad h \equiv h_{\alpha}^{\alpha} .
$$

From eq. (2.11) one finds

$$
\begin{aligned}
Q \bar{c}_{\mu} & =\frac{2}{\xi}\left[\partial^{\rho} A_{\rho} \circ A_{\mu}+2 \xi \partial_{\mu}(c \circ \bar{c})\right] \\
& =\frac{1}{\xi}\left[\partial^{\rho} \bar{h}_{\rho \mu}+\frac{\left(1+a_{1}\right)}{a_{3}} \partial_{\mu} \varphi\right],
\end{aligned}
$$

where we have used eq. (2.13). Comparing eqs. (2.14), (2.15), (2.16), we see that the only way for the gauge-fixing condition in gravity to correspond to the de Donder gauge, for arbitrary dilaton fields, is to set $a_{1}=-1$. Furthermore, the only effect of $a_{3}$ is to rescale the dilaton field, so we may set $a_{3}=1$ without loss of generality. Thus, we have finally obtained the dictionary

$$
h_{\mu \nu}=2 A_{\mu} \circ A_{\nu}-\eta_{\mu \nu}\left(A^{\rho} \circ A_{\rho}+2 \xi c \circ \bar{c}\right), \quad \varphi=\left(A^{\rho} \circ A_{\rho}+2 \xi c \circ \bar{c}\right) .
$$

Let us now apply this to the point charge in pure Yang-Mills theory, where the Lorenz gauge field is given $b y^{7}$

$$
A_{\mu}^{a}=\frac{g \alpha^{a}}{4 \pi r} u_{\mu}, \quad u_{\mu}=(1,0,0,0),
$$

where $\alpha^{a}$ is a colour vector, whose (proper-)time dependence may be neglected in the linearised theory (see e.g. ref. [17]). This satisfies the equation of motion

$$
\square A_{\mu}^{a}=j_{\mu}^{a}, \quad j_{\mu}^{a}=g \alpha^{a} \delta^{(3)}(\boldsymbol{x}) u_{\mu},
$$

where the current indeed represents a point-like colour charge located at the origin. One must also give the form of the (anti-)ghost fields $(c, \bar{c})$, which obey analogous equations of motion to eq. (2.19):

$$
\square c=j, \quad \square \bar{c}=\bar{j}, \quad j=g D \delta^{(3)}(\boldsymbol{x}), \quad \bar{j}=g \bar{D} \delta^{(3)}(\boldsymbol{x}),
$$

where $(D, \bar{D})$ are constant Grassmann numbers at linear order. It may at first seem strange that the ghost fields are being classically sourced, given that in scattering amplitudes they usually only occur in loops, where their job is to subtract unphysical degrees of freedom. However, we are here calculating the expectation value of a classical field, which is an off-shell quantity. It is thus gauge-variant, and the precise form it takes will depend on the choice of gauge-fixing, and hence the ghost contributions. Furthermore, we will see later

\footnotetext{
${ }^{6}$ In principle, we could have introduced a second arbitrary multiplier $\xi$ in eq. (2.14), but have instead chosen the same $\xi$ as eq. (2.4), which does not affect our conclusions.

${ }^{7}$ The form of the Lorenz gauge field is not unique, owing to the possibility of making residual gauge transformations that preserve the Lorenz gauge condition.
} 
on that the ghosts have a pivotal role to play when we take the double copy. Returning to the point charge, the solution of eq. (2.20) is straightforward:

$$
c=\frac{g D}{4 \pi r}, \quad \bar{c}=\frac{g \bar{D}}{4 \pi r},
$$

and we can now apply the dictionary of eq. (2.17) to double copy the above results to gravity. We first need the form of the spectator field that appears in the circle product of eq. (2.1). This should correspond to the inverse of the scalar propagator [28], which in the present (static) case reads ${ }^{8}$

$$
\Phi^{a a^{\prime}}=\frac{g \delta^{a a^{\prime}}}{4 \pi r} .
$$

To use eq. (2.17), we may take the Fourier transform $\mathcal{F}$ to momentum space, such that the convolutions are replaced by products. Using the simple result

$$
\mathcal{F}\left[\frac{1}{r}\right]=\frac{\delta\left(k^{0}\right)}{\boldsymbol{k}^{2}},
$$

where $k$ is the 4 -momentum variable conjugate to $x$, one finds

$$
\mathcal{F}\left[A_{\mu} \circ A_{\nu}\right]=\frac{g(\alpha \cdot \alpha) \delta\left(k^{0}\right)}{4 \pi \boldsymbol{k}^{2}} u_{\mu} u_{\nu}, \quad \mathcal{F}[c \circ \bar{c}]=\frac{g(D \cdot \bar{D}) \delta\left(k^{0}\right)}{4 \pi \boldsymbol{k}^{2}},
$$

and thus

$$
\bar{h}_{\mu \nu}=\frac{g}{4 \pi r}\left[2(\alpha \cdot \alpha) u_{\mu} u_{\nu}+2 \xi(D \cdot \bar{D}) \eta_{\mu \nu}\right], \quad \varphi=\frac{g}{4 \pi r}[\alpha \cdot \alpha+2 \xi D \cdot \bar{D}] .
$$

Some comments are in order. Firstly, we see that the choice of ghost sources $(D, \bar{D})$ completely specifies how the trace degree of freedom of the graviton, and the dilaton field, can be unambiguously disentangled from each other, at least in principle. This ambiguity has been noted in other approaches to the classical double copy [22]. Secondly, we may compare eq. (2.25) with the known form of the linearised JNW solution in the de Donder gauge (see e.g. ref. [22]):

$$
\bar{h}_{\mu \nu}=\frac{\kappa}{2} \frac{M}{4 \pi r} u_{\mu} u_{\nu}, \quad \varphi=-\frac{\kappa}{2} \frac{Y}{4 \pi r},
$$

where $M$ is the Schwarzschild mass term, and $Y$ an additional independent parameter. We then immediately see that the only way to recover the form of eq. (2.26) from eq. (2.25) is to choose the ghost sources to vanish i.e. $D=\bar{D}=0$. In that case, we do not recover the full two-parameter JNW solution, but only a special case. That is, upon replacing the coupling constants appropriately, and also the arbitrary colour charge by the Schwarzschild mass:

$$
g \rightarrow \frac{\kappa}{2}, \quad 2(\alpha \cdot \alpha) \rightarrow M
$$

\footnotetext{
${ }^{8}$ Note that we have here introduced a factor of the gauge theory coupling $g$ in the spectator field, for book-keeping purposes: the double copy dictionary then consists of obtaining gravity fields linear in $\kappa$ from gauge fields linear in $g$. This has an analogue in the Kerr-Schild double copy of ref. [4], in which the scalar field used to formulate the copy procedure contains a factor of the gauge theory coupling constant.
} 
we find that $Y=-M / 2$ is fixed, and thus not independent from $M$. That this is the correct outcome follows directly from the dictionary of eq. (2.17), which implies

$$
\bar{h}_{\mu \nu}=2\left[A_{\mu} \circ A_{\nu}+\xi \eta_{\mu \nu} c \circ \bar{c}\right] .
$$

We then see directly that one may only achieve the vanishing of the de Donder gauge constraint of eq. (2.15) upon imposing the Lorenz constraint on the gauge field by setting the (anti-)ghost fields to zero.

Some care is needed to interpret what has gone on here. It would not be correct, for example, to conclude that the double copy of the point charge is only a special case of JNW: this would directly contradict the results of refs. [4, 27]. Instead the problem can be traced back to the fact that we have demanded that the double copy dictionary of eqs. (2.2), (2.3) did not contain non-local derivative operators in position space. This choice has proven to be overly restrictive, and indeed insufficient to explore the full double copy of the point charge. As we will see in the following section, a very different situation occurs if the locality assumption is relaxed. However, it should also be stressed that the results of this section are also specific to having chosen the Lorenz and de Donder gauges in the gauge and gravity theory respectively - it may well be that a local dictionary can lead to more general JNW solutions if a different gauge is chosen.

\section{The JNW solution from a general dictionary}

The results of the previous section suggest that a more general dictionary is needed in order to relate gauge and graviton fields in the Lorenz and de Donder gauges. To this end, we may write the most general possible covariant ansatz for the graviton and dilaton containing gauge, (anti-)ghost fields and (inverse) derivative operators, as follows:

$$
\begin{aligned}
h_{\mu \nu}= & 2 A_{\mu} \circ A_{\nu}+\frac{d_{1}}{\square} \partial_{(\mu} A_{\nu)} \circ \partial A+\frac{d_{2}}{\square^{2}} \partial_{\mu} \partial_{\nu} \partial A \circ \partial A+d_{3} \frac{\partial_{\mu} \partial_{\nu}}{\square} A^{\rho} \circ A_{\rho}+d_{4} \frac{\partial_{\mu} \partial_{\nu}}{\square} c \circ \bar{c} \\
& +\eta_{\mu \nu}\left[a_{1} A^{\rho} \circ A_{\rho}+\frac{a_{2}}{\square} \partial A \circ \partial A+a_{3} c \circ \bar{c}\right] \\
\varphi= & b_{1} A^{\rho} \circ A_{\rho}+\frac{b_{2}}{\square} \partial A \circ \partial A+a_{3} c \circ \bar{c}
\end{aligned}
$$

where we use the slight shorthand notation $\partial A \equiv \partial \cdot A$. As in the previous section, imposing the BRST transformations of eq. (2.6) leads to constraints from the coefficient of $\eta_{\mu \nu}$ in the graviton, and also the dilaton, which in this case turn out to be

$$
a_{3}=2 \xi\left(a_{1}+a_{2}\right), \quad b_{3}=2 \xi\left(b_{1}+b_{2}\right) .
$$

One also finds expressions for the first-level gravitational ghost:

$$
c_{\mu}=\left(2+\frac{d_{1}}{2}\right) A_{\mu} \circ c+\left(\frac{d_{1}}{2}+d_{2}+d_{3}-\frac{d_{4}}{2 \xi}\right) \frac{\partial_{\mu}}{\square}(c \circ \partial A),
$$


where $\bar{c}_{\mu}$ is obtained by conjugation by replacing $c \rightarrow \bar{c}$. We can then impose the anti-ghost transformation of eq. (2.14) on the latter, which yields

$$
\begin{aligned}
\frac{1}{\xi} G_{\mu}[h]= & \left(2+\frac{d_{1}}{2}\right) \frac{1}{\xi} A_{\mu} \circ \partial A+\left(\frac{d_{1}}{2}+d_{2}+d_{3}-\frac{d_{4}}{2 \xi}\right) \frac{\partial_{\mu}}{\square} \partial A \circ \partial A \\
& +\left(2+d_{1}+d_{2}+d_{3}-\frac{d_{4}}{2 \xi}\right) \partial_{\mu}(c \circ \bar{c}) .
\end{aligned}
$$

However, we want the gauge-fixing condition in gravity to vanish when the Lorenz gauge condition $\partial A=0$ is satisfied in gauge theory, ${ }^{9}$ immediately implying

$$
d_{4}=2 \xi\left(2+d_{1}+d_{2}+d_{3}\right)
$$

which we may use to tidy up eq. (3.4):

$$
G_{\mu}[h]=\left(2+\frac{d_{1}}{2}\right)\left[A_{\mu} \circ \partial A-\frac{\partial_{\mu}}{\square} \partial A \circ \partial A\right] .
$$

Furthermore, we may substitute the constraints of eqs. (3.2), (3.5) into eq. (3.1) and derive, after some work,

$$
\begin{aligned}
\partial^{\nu} \bar{h}_{\mu \nu}= & \left(2+\frac{d_{1}}{2}\right) \partial A \circ A_{\mu}-\left(a_{2}-\frac{d_{2}}{2}\right) \frac{\partial_{\mu}}{\square} \partial A \circ \partial A-\left(1+a_{1}-\frac{d_{3}}{2}\right) \partial_{\mu} A^{\rho} \circ A_{\rho} \\
& -2 \xi\left(-1+a_{1}+a_{2}-\frac{d_{1}+d_{2}+d_{3}}{2}\right) \partial_{\mu} c \circ \bar{c}
\end{aligned}
$$

Equating this with eq. (3.6) yields the additional constraints

$$
a_{2}=2+\frac{d_{1}+d_{2}}{2}, \quad a_{1}=-1+\frac{d_{3}}{2},
$$

such that substituting these into eq. (3.1) yields the most general dictionary relating the Lorenz and de Donder fields:

$$
\begin{aligned}
h_{\mu \nu}= & 2 A_{\mu} \circ A_{\nu}+\frac{d_{1}}{\square} \partial_{(\mu} A_{\nu)} \circ \partial A+\frac{d_{2}}{\square^{2}} \partial_{\mu} \partial_{\nu} \partial A \circ \partial A+d_{3} \frac{\partial_{\mu} \partial_{\nu}}{\square} A^{\rho} \circ A_{\rho} \\
& +2 \xi\left(2+d_{1}+d_{2}+d_{3}\right) \frac{\partial_{\mu} \partial_{\nu}}{\square} c \circ \bar{c}+\eta_{\mu \nu}\left[\left(-1+\frac{d_{3}}{2}\right) A^{\rho} \circ A_{\rho}+\left(2+\frac{d_{1}+d_{2}}{2}\right) \frac{1}{\square} \partial A \circ \partial A\right. \\
& \left.+2 \xi\left(1+\frac{d_{1}+d_{2}+d_{3}}{2}\right) c \circ \bar{c}\right] ; \\
\varphi= & b_{1} A^{\rho} \circ A_{\rho}+\frac{b_{2}}{\square} \partial A \circ \partial A+2 \xi\left(b_{1}+b_{2}\right) c \circ \bar{c} .
\end{aligned}
$$

We thus see that the requirement that our two gauge choices match is insufficient to completely fix the dictionary, leaving a number of remaining arbitrary parameters. These represent the ability to make residual gauge transformations and field redefinitions that

\footnotetext{
${ }^{9}$ In previous works on the convolutional double copy, the BRST gauge mapping was enforced without requiring the gauge-fixing condition in gravity to vanish if its gauge theory counterpart does. Here we need to implement such conditions, given that we are considering explicit solutions.
} 
preserve the de Donder gauge condition. ${ }^{10}$ To examine the double copy of the point charge, it is sufficient to choose a particular case of the dictionary, and to this end we will choose

$$
d_{1}=-2, \quad d_{2}=d_{3}=0, \quad b_{1}=2, \quad b_{2}=0,
$$

leading to the simple dictionary

$$
\bar{h}_{\mu \nu}=2 A_{\mu} \circ A_{\nu}-\frac{2}{\square} \partial_{(\mu} A_{\nu)} \circ \partial A, \quad \varphi=2 A^{\rho} \circ A_{\rho}+4 \xi c \circ \bar{c} .
$$

Fourier transforming to momentum space and using the results of eqs. (2.18), (2.21), (2.22), one now finds that the graviton and dilaton fields are given by

$$
\bar{h}_{\mu \nu}=\frac{g(\alpha \cdot \alpha)}{4 \pi r} u_{\mu} u_{\nu}, \quad \varphi=\frac{g}{4 \pi r}[(\alpha \cdot \alpha)+4 \xi D \cdot \bar{D}] .
$$

We can recognise this as the full two-parameter JNW solution, provided that we identify

$$
g \rightarrow \frac{\kappa}{2}, \quad(\alpha \cdot \alpha) \rightarrow M, \quad[(\alpha \cdot \alpha)+4 g \xi D \cdot \bar{D}] \rightarrow-Y .
$$

The first and second of these replacements correspond to the usual replacement of couplings in the double copy, and colour charge by Schwarzschild mass [4]. The third replacement generates the coefficient of the dilaton in the JNW solution, which is then determined by the ghost sources. Thus, we see that the full two-parameter JNW solution is obtained, commensurate with refs. [22, 27]. Furthermore, the convolutional double copy provides a key insight into where these two parameters come from, namely the ability to source the ghosts and gauge fields (whilst keeping the Lorenz and de Donder gauges) independently. Indeed, the gravitational equations of motion $^{11}$

$$
\frac{1}{2} \square \bar{h}_{\mu \nu}=j_{\mu \nu}, \quad \square \varphi=j_{\varphi},
$$

together with eqs. (2.19), (2.20) (with sources left general), and the dictionary of eq. (3.11), imply the following relations between the sources in the gauge and gravity theory:

$$
\begin{aligned}
j_{\mu \nu} & =\frac{1}{\square} j_{\mu} \circ j_{\nu}-\frac{1}{\square^{2}} \partial_{(\mu} j_{\nu)} \circ \partial j, \\
j_{\varphi} & =\frac{2}{\square} j^{\rho} \circ j_{\rho}-\frac{4}{\square} j \circ \bar{j} .
\end{aligned}
$$

These relations directly encode the fact that independence of the graviton and dilaton sources are inherited from the separate gauge and (anti-)ghost sources in the single copy theory.

It is instructive to compare our results with those of ref. [22], which also considered how to obtain the JNW solution from the double copy. By applying a generalisation of the

\footnotetext{
${ }^{10}$ This is a consequence of the fact that the dictionary is invertible - at least at linear level. Indeed, imagine starting with the simplest dictionary [such as the one presented in $[25,48]$ ] — one can see that all the YM convolution products appearing with arbitrary parameters in (3.9) can be written as (possibly non-local) linear combinations of the graviton and dilaton as defined in $[25,48]$.

${ }^{11}$ We have here chosen $\xi=-1$ for simplicity, which does not affect our argument.
} 
BCJ double copy to perturbative solutions of the equation of motion, the authors defined a fat graviton field $H_{\mu \nu}$, satisfying the linearised equation of motion

$$
\square H_{\mu \nu}=0,
$$

and which combines the physical degrees of freedom of the graviton, dilaton and axion. One must then provide a prescription for obtaining the individual fields (in a particular generalised gauge) from the fat graviton. To this end, ref. [22] used the ansatz (at linearised level)

$$
H_{\mu \nu}=\bar{h}_{\mu \nu}+B_{\mu \nu}+P_{\mu \nu}^{q}(\varphi-\bar{h})
$$

where $\bar{h}=\bar{h}_{\mu}^{\mu}$, and we have defined the projection operator

$$
P_{\mu \nu}^{q}=\frac{1}{d-2}\left(\eta_{\mu \nu}-\frac{q_{\mu} \partial_{\nu}+q_{\nu} \partial_{\mu}}{q \cdot \partial}\right)
$$

where $q$ is a constant null 4 -vector such that $q \cdot k \neq 0$, if $k$ is the momentum of the fat graviton. The individual fields (referred to as skinny fields in ref. [22]) are given by

$$
\begin{aligned}
\bar{h}_{\mu \nu} & =\frac{1}{2}\left(H_{\mu \nu}+H_{\nu \mu}\right)-P_{\mu \nu}^{q} H, \\
B_{\mu \nu} & =\frac{1}{2}\left(H_{\mu \nu}-H_{\nu \mu}\right), \\
\varphi & =H,
\end{aligned}
$$

where one may show that the graviton thus obtained is in de Donder gauge. The role of the projection operator of eq. (3.18) is to resolve the ambiguity regarding how to divide the trace of the fat graviton $(H)$ between the graviton trace $(\bar{h})$ and dilaton $(\varphi)$ degrees of freedom. In eq. (3.19), all of $H$ is taken to correspond to the dilaton, although it is possible to mix up $\varphi$ and $\bar{h}$ by performing residual gauge transformations that preserve the de Donder gauge condition. Using this approach, ref. [22] showed that the most general fat graviton for the JNW solution can be written as

$$
H_{\mu \nu}=\frac{\kappa}{2} \frac{1}{4 \pi r}\left(M u_{\mu} u_{\nu}+(M-Y) \frac{1}{2}\left(\eta_{\mu \nu}-q_{\mu} l_{\nu}-q_{\nu} l_{\mu}\right)\right),
$$

where $u_{\mu}$ has been defined above, and $l_{\mu}$ is a 4 -vector such that $q \cdot l=1$. This reproduces the de Donder gauge graviton and dilaton fields of eq. (2.26), and is furthermore such that the single copy is always a gauge theory point charge. Furthermore, ref. [22] showed how eq. (3.20) could be used as a building block in understanding the classical double copy at higher perturbative orders.

It is interesting to note a number of similarities between the convolutional double copy and fat graviton approaches. Firstly, they both produce the full two-parameter JNW solution in the gravity theory. In the convolutional approach, this manifests itself in the freedom to source the ghosts independently from the gauge fields, while in the approach of ref. [22], this comes from the ability to redefine the coefficient of the projection operator (amounting to superposing arbitrary amounts of the linearised dilaton and graviton solutions). Both approaches have something in common with the double copy for scattering 
amplitudes, in which the external degrees of freedom (graviton, dilaton or axion) appearing in a gravity amplitude depend on the physical polarisation states one chooses in the two gauge theory amplitudes entering the double copy.

A second similarity between the convolutional and fat graviton approaches concerns the use of non-local operators in position space. In eq. (3.19), the projector $P_{\mu \nu}^{q}$ is nonlocal, as can be seen from its definition in eq. (3.18). This mirrors the use of non-local terms in the convolutional dictionary of eq. (3.1), in order to be able to obtain the full JNW solution rather than a special case. There may, of course, be choices of gauge in both approaches such that non-local terms are not needed, but it is nevertheless intriguing that non-locality arises whatever method one chooses for performing the double copy to a de Donder gauge solution.

\section{Conclusion}

In this paper, we have examined a particular approach for obtaining linearised solutions of gravity theories as a double copy of gauge theory, namely the convolutional approach of refs. [28-33, 47, 48, 51, 52]. Our aim was twofold. Firstly, it is instructive to clarify how to use this approach by looking at particular solutions. Secondly, by choosing the point charge in pure Yang-Mills theory, the convolutional approach offers a useful complementary point of view to related approaches, that have debated whether the full JNW solution in $\mathcal{N}=0$ supergravity - or a special case of this - is the appropriate gravity solution [4, 18, 22, 27].

To use the convolutional double copy, one must posit a suitable dictionary between the gauge and gravity fields, containing free parameters. The latter can then be at least partially fixed by imposing the correct BRST transformations in both theories, which explicitly brings in the gauge-fixing conditions. Unfixed parameters correspond to residual generalised gauge transformations that leave the gauge-fixing condition unchanged. In examining the point charge in the Lorenz gauge, we found that a completely local dictionary was insufficient to obtain the full two-parameter JNW solution in the gravity theory in de Donder gauge, as seen in the previous results of refs. [22, 27]. The solution to this problem was to include non-local operators in position space, which indeed allowed us to obtain the full JNW solution. An arbitrary combination of $M$ and $Y$ in this solution can be made by choosing sources for the BRST ghost fields appropriately, mirroring the role of the projection operator in ref. [22]. Furthermore, many free parameters in the general dictionary remained unconstrained, indicating that the double copy to de Donder gauge is robust under a non-trivial group of generalised gauge transformations.

There are many possible avenues for further work. It would be interesting, for example, to relate the convolutional approach to the Kerr-Schild approach of ref. [4]. Although this seems a natural thing to do - given that the Kerr-Schild procedure linearises the field equations on both sides of the double copy - it is not immediately clear how to proceed. Not all metrics, for example, can be expressed in Kerr-Schild coordinates, so that there is no such thing as a general "Kerr-Schild gauge". Without an explicit gauge-fixing term, it is difficult to unambiguously apply the BRST procedure to relate the gauge and gravity theories. Another important development would be to extend the convolutional double copy 
in the BRST context to non-linear orders in perturbation theory. This could provide key insights into obtaining pure gravity perturbation theory from the double copy, given that the convolution approach makes clear how the dilaton is generated (or not) in the gravity theory, and can also be used in arbitrary gauges. Work in these and other directions is in progress [25].

\section{Acknowledgments}

We thank Leron Borsten and Donal O'Connell for useful conversations. This work has been supported by the U.K. Science and Technology Facilities Council (STFC) Consolidated Grant ST/P000754/1 "String theory, gauge theory and duality", and by the European Union Horizon 2020 research and innovation programme under the Marie SkłodowskaCurie grant agreement No. 764850 "SAGEX". AL is supported in part by the Department of Energy under Award Number DESC000993. SN is supported by STFC grant ST/P000703/1 and a Leverhulme Research Project Grant. This research was supported by the Munich Institute for Astro- and Particle Physics (MIAPP) which is funded by the Deutsche Forschungsgemeinschaft (DFG, German Research Foundation) under Germany's Excellence Strategy — EXC-2094 - 390783311.

Open Access. This article is distributed under the terms of the Creative Commons Attribution License (CC-BY 4.0), which permits any use, distribution and reproduction in any medium, provided the original author(s) and source are credited.

\section{References}

[1] Z. Bern, J.J.M. Carrasco and H. Johansson, New Relations for Gauge-Theory Amplitudes, Phys. Rev. D 78 (2008) 085011 [arXiv: 0805.3993] [INSPIRE].

[2] Z. Bern, J.J.M. Carrasco and H. Johansson, Perturbative Quantum Gravity as a Double Copy of Gauge Theory, Phys. Rev. Lett. 105 (2010) 061602 [arXiv:1004.0476] [INSPIRE].

[3] Z. Bern, T. Dennen, Y.-t. Huang and M. Kiermaier, Gravity as the Square of Gauge Theory, Phys. Rev. D 82 (2010) 065003 [arXiv: 1004.0693] [inSPIRE].

[4] R. Monteiro, D. O'Connell and C.D. White, Black holes and the double copy, JHEP 12 (2014) 056 [arXiv: 1410.0239] [INSPIRE].

[5] A. Luna, R. Monteiro, D. O'Connell and C.D. White, The classical double copy for Taub-NUT spacetime, Phys. Lett. B 750 (2015) 272 [arXiv:1507.01869] [INSPIRE].

[6] A. Luna, R. Monteiro, I. Nicholson, D. O'Connell and C.D. White, The double copy: Bremsstrahlung and accelerating black holes, JHEP 06 (2016) 023 [arXiv:1603.05737] [INSPIRE].

[7] N. Bahjat-Abbas, A. Luna and C.D. White, The Kerr-Schild double copy in curved spacetime, JHEP 12 (2017) 004 [arXiv: 1710.01953] [INSPIRE].

[8] D.S. Berman, E. Chacón, A. Luna and C.D. White, The self-dual classical double copy, and the Eguchi-Hanson instanton, JHEP 01 (2019) 107 [arXiv: 1809.04063] [INSPIRE]. 
[9] A.K. Ridgway and M.B. Wise, Static Spherically Symmetric Kerr-Schild Metrics and Implications for the Classical Double Copy, Phys. Rev. D 94 (2016) 044023 [arXiv: 1512.02243] [INSPIRE].

[10] M. Carrillo-González, R. Penco and M. Trodden, The classical double copy in maximally symmetric spacetimes, JHEP 04 (2018) 028 [arXiv:1711.01296] [INSPIRE].

[11] M. Carrillo González, B. Melcher, K. Ratliff, S. Watson and C.D. White, The classical double copy in three spacetime dimensions, JHEP 07 (2019) 167 [arXiv:1904.11001] [INSPIRE].

[12] N. Bahjat-Abbas, R. Stark-Muchão and C.D. White, Monopoles, shockwaves and the classical double copy, JHEP 04 (2020) 102 [arXiv: 2001.09918] [INSPIRE].

[13] L. Alfonsi, C.D. White and S. Wikeley, Topology and Wilson lines: global aspects of the double copy, JHEP 07 (2020) 091 [arXiv: 2004.07181] [INSPIRE].

[14] R. Alawadhi, D.S. Berman, B. Spence and D. Peinador Veiga, S-duality and the double copy, JHEP 03 (2020) 059 [arXiv: 1911.06797] [INSPIRE].

[15] R. Monteiro, I. Nicholson and D. O'Connell, Spinor-helicity and the algebraic classification of higher-dimensional spacetimes, Class. Quant. Grav. 36 (2019) 065006 [arXiv:1809.03906] [INSPIRE].

[16] A. Luna, R. Monteiro, I. Nicholson and D. O'Connell, Type D Spacetimes and the Weyl Double Copy, Class. Quant. Grav. 36 (2019) 065003 [arXiv:1810.08183] [InSPIRE].

[17] W.D. Goldberger and A.K. Ridgway, Radiation and the classical double copy for color charges, Phys. Rev. D 95 (2017) 125010 [arXiv:1611.03493] [InSPIRE].

[18] W.D. Goldberger, S.G. Prabhu and J.O. Thompson, Classical gluon and graviton radiation from the bi-adjoint scalar double copy, Phys. Rev. D 96 (2017) 065009 [arXiv:1705.09263] [INSPIRE].

[19] W.D. Goldberger and A.K. Ridgway, Bound states and the classical double copy, Phys. Rev. D 97 (2018) 085019 [arXiv: 1711.09493] [InSPIRE].

[20] W.D. Goldberger, J. Li and S.G. Prabhu, Spinning particles, axion radiation, and the classical double copy, Phys. Rev. D 97 (2018) 105018 [arXiv:1712.09250] [INSPIRE].

[21] W.D. Goldberger and J. Li, Strings, extended objects, and the classical double copy, JHEP 02 (2020) 092 [arXiv: 1912.01650] [INSPIRE].

[22] A. Luna et al., Perturbative spacetimes from Yang-Mills theory, JHEP 04 (2017) 069 [arXiv: 1611.07508] [INSPIRE].

[23] A. Luna, I. Nicholson, D. O'Connell and C.D. White, Inelastic Black Hole Scattering from Charged Scalar Amplitudes, JHEP 03 (2018) 044 [arXiv: 1711.03901] [INSPIRE].

[24] B. Maybee, D. O'Connell and J. Vines, Observables and amplitudes for spinning particles and black holes, JHEP 12 (2019) 156 [arXiv: 1906.09260] [INSPIRE].

[25] L. Borsten and S. Nagy, The pure BRST Einstein-Hilbert Lagrangian from the double-copy to cubic order, JHEP 07 (2020) 093 [arXiv: 2004.14945] [INSPIRE].

[26] K. Lee, Kerr-Schild Double Field Theory and Classical Double Copy, JHEP 10 (2018) 027 [arXiv: 1807.08443] [INSPIRE].

[27] K. Kim, K. Lee, R. Monteiro, I. Nicholson and D. Peinador Veiga, The Classical Double Copy of a Point Charge, JHEP 02 (2020) 046 [arXiv: 1912.02177] [INSPIRE]. 
[28] A. Anastasiou, L. Borsten, M.J. Duff, L.J. Hughes and S. Nagy, Yang-Mills origin of gravitational symmetries, Phys. Rev. Lett. 113 (2014) 231606 [arXiv:1408.4434] [INSPIRE].

[29] A. Anastasiou et al., Twin supergravities from Yang-Mills theory squared, Phys. Rev. D 96 (2017) 026013 [arXiv:1610.07192] [inSPIRE].

[30] G.L. Cardoso, S. Nagy and S. Nampuri, A double copy for $\mathcal{N}=2$ supergravity: a linearised tale told on-shell, JHEP 10 (2016) 127 [arXiv:1609.05022] [INSPIRE].

[31] G. Cardoso, S. Nagy and S. Nampuri, Multi-centered $\mathcal{N}=2$ BPS black holes: a double copy description, JHEP 04 (2017) 037 [arXiv:1611.04409] [INSPIRE].

[32] A. Anastasiou, L. Borsten, M.J. Duff, A. Marrani, S. Nagy and M. Zoccali, Are all supergravity theories Yang-Mills squared?, Nucl. Phys. B 934 (2018) 606 [arXiv:1707.03234] [INSPIRE].

[33] A. Anastasiou, L. Borsten, M.J. Duff, A. Marrani, S. Nagy and M. Zoccali, The Mile High Magic Pyramid, Contemp. Math. 721 (2019) 1 [arXiv:1711.08476] [INSPIRE].

[34] L. Borsten, M.J. Duff, L.J. Hughes and S. Nagy, Magic Square from Yang-Mills Squared, Phys. Rev. Lett. 112 (2014) 131601 [arXiv:1301.4176] [INSPIRE].

[35] A. Anastasiou, L. Borsten, M.J. Duff, L.J. Hughes and S. Nagy, A magic pyramid of supergravities, JHEP 04 (2014) 178 [arXiv:1312.6523] [INSPIRE].

[36] A. Anastasiou, L. Borsten, M.J. Hughes and S. Nagy, Global symmetries of Yang-Mills squared in various dimensions, JHEP 01 (2016) 148 [arXiv:1502.05359] [INSPIRE].

[37] Z. Bern, J.J. Carrasco, W.-M. Chen, H. Johansson and R. Roiban, Gravity Amplitudes as Generalized Double Copies of Gauge-Theory Amplitudes, Phys. Rev. Lett. 118 (2017) 181602 [arXiv: 1701.02519] [INSPIRE].

[38] Z. Bern, J.J.M. Carrasco, W.-M. Chen, H. Johansson, R. Roiban and M. Zeng, Five-loop four-point integrand of $N=8$ supergravity as a generalized double copy, Phys. Rev. D 96 (2017) 126012 [arXiv:1708.06807] [INSPIRE].

[39] H. Kawai, D.C. Lewellen and S.H.H. Tye, A Relation Between Tree Amplitudes of Closed and Open Strings, Nucl. Phys. B 269 (1986) 1 [inSPIRE].

[40] L.D. Faddeev and V.N. Popov, Feynman Diagrams for the Yang-Mills Field, Phys. Lett. B 25 (1967) 29 [INSPIRE].

[41] C. Becchi, A. Rouet and R. Stora, The Abelian Higgs-Kibble Model. Unitarity of the $S$ Operator, Phys. Lett. B 52 (1974) 344 [INSPIRE].

[42] C. Becchi, A. Rouet and R. Stora, Renormalization of the Abelian Higgs-Kibble Model, Commun. Math. Phys. 42 (1975) 127 [inSPIRE].

[43] C. Becchi, A. Rouet and R. Stora, Renormalization of Gauge Theories, Annals Phys. 98 (1976) 287 [INSPIRE].

[44] I.V. Tyutin, Gauge Invariance in Field Theory and Statistical Physics in Operator Formalism, arXiv:0812.0580 [INSPIRE].

[45] E.S. Fradkin and G.A. Vilkovisky, Quantization of relativistic systems with constraints, Phys. Lett. B $5 \mathbf{5}$ (1975) 224 [INSPIRE].

[46] I.A. Batalin and G.A. Vilkovisky, Relativistic S Matrix of Dynamical Systems with Boson and Fermion Constraints, Phys. Lett. B 69 (1977) 309 [INSPIRE]. 
[47] A. Anastasiou, L. Borsten, M.J. Duff, S. Nagy and M. Zoccali, Gravity as Gauge Theory Squared: A Ghost Story, Phys. Rev. Lett. 121 (2018) 211601 [arXiv:1807.02486] [INSPIRE].

[48] L. Borsten, I. Jubb, V. Makwana and S. Nagy, Gauge $\times$ gauge on spheres, JHEP 06 (2020) 096 [arXiv: 1911.12324] [INSPIRE].

[49] W. Siegel, Superstrings give old minimal supergravity, Phys. Lett. B 211 (1988) 55 [INSPIRE].

[50] W. Siegel, Curved extended superspace from Yang-Mills theory a la strings, Phys. Rev. D 53 (1996) 3324 [hep-th/9510150] [INSPIRE].

[51] G. Lopes Cardoso, G. Inverso, S. Nagy and S. Nampuri, Comments on the double copy construction for gravitational theories, PoS CORFU2017 (2018) 177 [arXiv:1803.07670] [INSPIRE].

[52] L. Borsten, Gravity as the square of gauge theory: a review. Riv. Nuovo Cim. 43 (2020) 97 [INSPIRE].

[53] A.I. Janis, E.T. Newman and J. Winicour, Reality of the Schwarzschild Singularity, Phys. Rev. Lett. 20 (1968) 878 [INSPIRE].

[54] C.D. White, Exact solutions for the biadjoint scalar field, Phys. Lett. B 763 (2016) 365 [arXiv: 1606.04724] [INSPIRE].

[55] P.-J. De Smet and C.D. White, Extended solutions for the biadjoint scalar field, Phys. Lett. $B \mathbf{7 7 5}$ (2017) 163 [arXiv:1708.01103] [INSPIRE].

[56] N. Bahjat-Abbas, R. Stark-Muchão and C.D. White, Biadjoint wires, Phys. Lett. B 788 (2019) 274 [arXiv: 1810.08118] [INSPIRE]. 